\title{
Segmentation of Bone Marrow Biopsies by Mathematical Morphology in Color Spaces
}

\author{
J. Pastore, A. Bouchet, E. Moler and V. Ballarin
}

\begin{abstract}
Anatomic and pathologic reports of histological slides give results indicating the presence of trabeculae, hematopoietic and fat cells. This allows evaluating the grade of some metabolic disorder, comparing normal values with pathological ones. Generally, quantifications are carried out by simple visual inspection presenting big inter-and intra-user differences. This work presents an automatic method for detecting different types of tissues in bone marrow biopsies using Mathematical Morphology in color spaces, in order to determine the marrow cellularity accurately. The method was tested with 30 images of bone marrow biopsies presenting different distributions of cellularity.
\end{abstract}

Keywords- Bone Marrow Biopsies, Segmentation, Mathematical Morphology, Color Spaces.

\section{INTRODUCCIÓN}

$\mathrm{L}$ OS INFORMES de biopsias de médula ósea entregan resultados porcentuales de la presencia de trabéculas, células adiposas y hematopoyéticas. Dichos porcentajes se toman como criterio genérico mediante el cual se pueden determinar, entre otros factores, diversos desórdenes metabólicos [1]. Sin embargo, no es un problema sencillo realizar una correcta identificación de la estructura trabecular, dado que los colores que la representan son muy similares a los de las regiones de celularidad [2].

Por lo tanto, es de interés contar con una herramienta que, a través de técnicas del Procesamiento Digital de Imágenes (PDI), contribuya a la cuantificación de las estructuras trabeculares presentes, para realizar cálculos posteriores $\mathrm{y}$ obtener diagnósticos [3].

La morfología de las trabéculas, médula ósea y tejido adiposo, es absolutamente irregular. En una escena se observan tramas, formas circulares, elipsoides, entre otras, pero ninguna descriptible por formas geométricas determinadas. Esto hace que los cálculos de la geometría analítica para el cálculo de las medidas sean dificultosos de aplicar. Es aquí donde el PDI

tiene un amplio campo de aplicación. El PDI estudia las

J. I. Pastore, CONICET - Universidad Nacional de Mar del Plata, Mar del Plata, Argentina, jpastore@fi.mdp.edu.ar

A. Bouchet, CONICET - Universidad Nacional de Mar del Plata, Mar del Plata, Argentina, abouchet@fi.mdp.edu.ar

E. Moler, Universidad Nacional de Mar del Plata, Mar del Plata, Argentina, emoler@fi.mdp.edu.ar

V. Ballarin, Universidad Nacional de Mar del Plata, Mar del Plata, Argentina, vballari@fi.mdp.edu.ar técnicas de transformación, manipulación y extracción de información de imágenes digitales. Dichas técnicas se utilizan para analizar, realzar, restaurar, modificar o cuantificar imágenes digitales para múltiples aplicaciones [4].

Entre los diversos campos de aplicación, el diagnóstico médico por imágenes despierta interés en especialistas tanto de medicina como de procesamiento. La cantidad de usuarios de estas herramientas supera ampliamente los avances en esta disciplina. Sin embargo, el software existente, no siempre atiende completamente las demandas. Más aún, cada requerimiento implica el estudio de nuevas técnicas y su implementación computacional para una aplicación satisfactoria. Con el advenimiento de las técnicas de PDI, calcular medidas en imágenes provenientes de cortes histológicos se solucionan en gran medida, ya que al considerar a la imagen como una matriz de puntos, se pueden realizar mediciones de áreas, perímetros y fórmulas derivadas con una gran precisión utilizando matemática discreta.

Sin embargo, el problema reside en que dada la variabilidad de las escenas a estudiar es difícil desarrollar un software que permita realizar estos procesos en forma automática. Se deben realizar exhaustivos pre-procesamientos de la imagen a analizar, tarea que en algunos casos requiere de conocimientos técnicos específicos. Esta situación es la que contribuye en forma negativa para que esta herramienta sea adoptada mayoritariamente y se sigan utilizando técnicas manuales.

Una de las tareas del PDI, que puede determinar el eventual éxito o fracaso de las mediciones, es la de separar previamente los objetos de interés. Esta etapa se denomina segmentación y juega un importante papel en muchas aplicaciones de imágenes donde se requieren delinear estructuras anatómicas y/o zonas de interés [5,6]. En este tipo de imágenes éste es un proceso complejo ya que dichas estructuras anatómicas no se encuentran perfectamente delimitadas. No existe, hasta el momento, un único método de segmentación que entregue resultados aceptables para todo tipo de imágenes médicas. La selección de un método apropiado de segmentación es una verdadera dificultad.

En este trabajo se presenta un método automático de detección de trabéculas en biopsias de médula ósea, utilizando Morfología Matemática Color, para su posterior cuantificación.

En las secciones siguientes se describen las principales características de las técnicas de PDI utilizadas y el método propuesto que permite la segmentación esperada. 


\section{MORFOLOGÍA MATEMÁTICA COLOR}

\section{A. Morfología Matemática Color}

La Morfología Matemática (MM) es una teoría basada en conceptos de geometría, álgebra y teoría de conjuntos. Originalmente fue propuesta para caracterizar propiedades físicas y estructurales de diversos materiales [7-9]. Estudia, mediante operaciones no lineales, las estructuras geométricas de las componentes presentes en una imagen. Esta teoría permite analizar la forma, tamaño, orientación y superposición de objetos. $\mathrm{Su}$ aspecto clave reside en el elemento estructurante (EE), un conjunto completamente definido y de geometría conocida, que es comparado con la imagen a partir de traslaciones. La forma, tamaño e intensidad del EE permiten testear y cuantificar de qué manera dicho elemento se encuentra o no, contenido en la imagen [9].

Las operaciones básicas de la MM son la erosión y la dilatación. Mediante la composición de estas operaciones es posible construir nuevos operadores como, por ejemplo, la apertura y el cierre [10].

Una imagen en niveles de gris puede ser modelada como una función $f: D_{f} \subset \square^{2} \rightarrow \square 0,255 \square$. Considerando el conjunto $\square 0,255 \square$ con el orden relativo al conjunto de los números reales, es fácil verificar que el conjunto de imágenes en niveles de gris constituye un reticulado completo pudiéndose definir las operaciones básica [11].

Sean $f$ y $g$ dos imágenes en niveles de gris, con dominios $D_{f}$ y $D_{g}$ respectivamente. La dilatación de la imagen $f$ por el EE $g$, notada por $\delta_{g}(f)$, se define como [7,8]:

$$
\delta_{g}(f)_{(s, t)}=\max _{(s-x, t-y) \in D_{i} ;(x, y) \in D_{s}}\{f(s-x, t-y)+g(x, y)\}
$$

La erosión de la imagen $f$ por el EE $g$, notada por $\varepsilon_{g}(f)$, se define como $[7,8]$ :

$$
\varepsilon_{g}(f)_{(s, t)}=\min _{(s+x, t+y) \in D_{i} ;(x, y) \in D_{x}}\{f(s+x, t+y)-g(x, y)\}
$$

Si el EE es plano, es decir $g(x, y)=0 \forall(x, y) \in D_{g}$, las ecuaciones (1) y (2) pueden escribirse de la siguiente manera:

$$
\begin{aligned}
& \delta_{g}(f)_{(s, t)}=\max _{(s-x, t-y) \in D_{l} ;(x, y) \in D_{g}}\{f(s-x, t-y)\} \\
& \varepsilon_{g}(f)_{(s, t)}=\min _{(s+x, t+y) \in D_{t} ;(x, y) \in D_{g}}\{f(s+x, t+y)\}
\end{aligned}
$$

Mediante la combinación de las operaciones básicas, pueden definirse los filtros morfológicos básicos apertura y cierre como se muestra a continuación.

La apertura morfológica de una imagen $f$ por el EE $g$ es definida por [7]:

$$
\gamma_{g}(f)=\delta_{g}\left(\varepsilon_{g}(f)\right)
$$

La apertura es de utilidad para eliminar detalles luminosos pequeños en relación al EE, quedando el resto de la imagen relativamente $\sin$ modificaciones.
El cierre morfológico de una imagen en niveles de gris $f$ por el EE $g$ es definido por [7]:

$$
\phi_{g}(f)=\varepsilon_{g}\left(\delta_{g}(f)\right)
$$

El cierre es de utilidad para eliminar detalles oscuros pequeños en relación al $\mathrm{EE}$, quedando el resto de la imagen relativamente sin modificaciones.

Dos operadores morfológicos ampliamente utilizados para eliminar el ruido presente en una imagen binaria son el area opening y el area closing, los cuales se definen a continuación.

El operador morfológico area opening, notado por $\gamma_{\lambda}^{\text {area }}$, de parámetro $\lambda$ sobre la imagen $A$ queda definido como [7]:

$$
\gamma_{\lambda}^{\text {area }}(A)=\left\{x \in A / \operatorname{Area}\left(C_{x}(A)\right) \geq \lambda\right\}
$$

Este operador elimina aquellas regiones conectadas de la imagen con área menor a $\lambda$.

El area closing, notado por $\phi_{\lambda}^{\text {area }}$, de parámetro $\lambda$ sobre la imagen $A$ queda definido como [7]:

$$
\phi_{\lambda}^{\text {area }}(A)=\left[\gamma_{\lambda}^{\text {area }}\left(A^{c}\right)\right]^{c}
$$

donde $A^{c}$ es el complemento de la imagen. Dicho operador elimina aquellas componentes conectadas con el fondo con un área menor a $\lambda$.

A pesar de que la MM está bien definida para imágenes binarias y en niveles de gris, no existe, en general, una extensión natural que permita definir los operadores básicos de esta metodología para ser aplicados en el dominio de imágenes color. A continuación se presenta una forma de abordar este problema basándose en la definición de un reticulado completo bajo ciertas características especiales.

Una imagen en color puede ser modelada como una función $f: D_{f} \subset \square^{2} \rightarrow \mathfrak{I} \subset \square^{3}$ donde $\mathfrak{I}$ representa un espacio de color. El procesamiento morfológico de imágenes en color suele hacerse desde dos puntos de vista: el procesamiento marginal y el procesamiento vectorial. El procesamiento marginal consiste en aplicar los operadores morfológicos definidos para imágenes en niveles de gris a cada una de las componentes cromática de la imagen. En el tratamiento vectorial se utiliza la teoría de reticulado completo y por lo tanto es necesario definir un orden sobre el subconjunto $\mathfrak{I}$ de $\square^{3}$. Como no se tiene un orden natural en estos conjuntos, es necesario dar una solución ad hoc, es decir, establecer un orden apropiado sobre el espacio de color $\mathfrak{I}$.

Sea $\mathfrak{I} \subset \square^{3}$ un espacio de color $\mathrm{y} \leq_{\mathfrak{I}}$ un orden que dota a $\mathfrak{I}$ de una estructura de reticulado completo. El espacio de funciones $f: D_{f} \subset \square^{2} \rightarrow \mathfrak{I} \subset \square^{3}$ con el orden $\leq$ definido como: $f, g: D \subset \square^{2} \rightarrow \mathfrak{I} \subset \square^{3}, \quad f \leq g \quad$ si $\quad$ y sólo sí $f(x) \leq_{\mathfrak{S}} g(x) \forall x \in D$, tiene también estructura de reticulado. Esto permite definir las operaciones básicas erosión y dilatación de una imagen color $f: D_{f} \subset \square^{2} \rightarrow \mathfrak{I} \subset \square^{3}$ por un elemento estructurante $B$ : 


$$
\begin{array}{r}
\varepsilon_{B}^{\leq_{3}}(f)=\inf _{s \in B}^{\leq_{3}}\left\{f \circ \tau_{s}\right\} \\
\delta_{B}^{\leq_{3}}(f)=\sup _{s \in B}^{\leq_{3}}\left\{f \circ \tau_{-s}\right\}
\end{array}
$$

siendo $\tau_{s}: \square^{2} \rightarrow \square^{2}$ la función traslación según el elemento $s \in \square^{2} \tau_{s}(x)=x+s$.

Es importante tener presente que la erosión y dilatación de una imagen en color dependerá fuertemente del orden establecido en el espacio cromático, por lo tanto, no tienen un único significado como en el caso de imágenes binarias e imágenes en niveles de gris. Si el orden establecido no es un orden total, el ínfimo y el supremo no necesariamente son mínimo y máximo respectivamente, pudiendo aparecer falsos colores en la imagen resultado de aplicar una erosión o dilatación, es decir, colores que no estaban en la imagen original. Cuando el orden fijado en el espacio cromático es total los ínfimos y supremos son mínimos y máximos respectivamente, y por lo tanto no aparecen falsos colores al aplicar las operaciones básicas.

Para este trabajo se utilizó el espacio cromático RGB con el orden lexicográfico o de diccionario. Este orden es un orden total y se define [12]:

$$
\left(r_{1}, g_{1}, b_{1}\right) \leq\left(r_{2}, g_{2}, b_{2}\right) \text { si }\left\{\begin{array}{l}
r_{1}<r_{2} \\
o \\
r_{1}=r_{2} y g_{1}<g_{2} \\
o \\
r_{1}=r_{2} \text { y } g_{1}=g_{2} \text { y } b_{1}<b_{2}
\end{array}\right.
$$

De la definición se desprende que el orden lexicográfico ordena los vectores a partir de una componente elegida como prioritaria (en la ecuación (11) la prioridad está en la primer componente, es decir, la componente Roja). Es evidente que la componente elegida como prioritaria tendrá más peso o importancia que otra y el orden dependerá de esta elección.

Una vez definidos los operadores básicos en el dominio de imágenes color se pueden definir los filtros morfológicos apertura y cierre como se hizo en las ecuaciones (5) y (6):

La apertura morfológica de una imagen color $f$ por el EE $B$ es definida por:

$$
\gamma_{B}^{\leq_{3}}(f)=\delta_{B}^{\leq_{3}}\left(\varepsilon_{B}^{\leq_{3}}(f)\right)
$$

El cierre morfológico de una imagen en niveles de gris $f$ por el EE $B$ es definido por:

$$
\phi_{B}^{\leq_{3}}(f)=\varepsilon_{B}^{\leq_{3}}\left(\delta_{B}^{\leq_{3}}(f)\right)
$$

Definidos los conceptos teóricos necesarios, en la siguiente sección se presenta el método propuesto.

\section{MATERIALES Y MÉTODOS}

\section{A. Materiales}

El material fue procesado con las siguientes especificaciones:

- Las muestras se fijaron en líquido de Bouin durante 24 hs. (ó 48 hs., según los casos), procediendo a su descalcificación y a su inclusión en parafina.
- Los cortes histológicos fueron realizados en un micrótomo rotativo con un espesor de $4 \mu \mathrm{m}$.

- Las tinciones histológicas utilizadas fueron: HematoxilinaEosina, como técnica de rutina, coloraciones para Fibras Reticulares (reticulina de Gomori), coloraciones para hierro (Perls) y coloración de Giemsa.

Para este trabajo se estudiaron treinta (30) imágenes. Éstas se obtuvieron de un Microscopio Óptico Medilux-12 con un objetivo 4X Plano 4X, N.A. 0.10 digitalizadas a través de una cámara color CCD Hitachi KP-C550. Esta cámara tiene una dimensión eficaz de $682(\mathrm{H})$ x 492 (V) píxeles y un rango de longitud de onda de 400 a $700 \mathrm{~nm}$. Soporta una resolución de video de 430 líneas, suficientes para capturar imágenes de las biopsias de 640 × 480 píxeles en una PC. Después de seleccionar el área de interés en el microscopio se adquiere una única imagen en cada caso.

\section{B. Método Propuesto}

El algoritmo desarrollado se dividió en las siguientes etapas:

Etapa 1: Filtrado morfológico mediante el operador Cierre definido para imágenes color con EE en forma de cruz.

Etapa 2: La imagen RGB obtenida en el paso anterior se descompone en sus tres componente Rojo, Verde y Azul. Sobre la componente Verde se aplica un procesamiento morfológico.

Etapa 3: Se realiza un realce en el espacio color HSI.

A continuación se detalla cada una de las etapas que conforman el algoritmo desarrollado:

Etapa 1: En primer lugar se aplica a la imagen original un Cierre definido para imágenes color con EE en forma de cruz con el objetivo de homogeneizar el tejido trabecular (Ver Fig. 1(b)).

Etapa 2: La imagen RGB obtenida en el paso anterior se descompone en sus tres componente Rojo, Verde y Azul. Se binariza la componente verde (Ver Fig. 1(c)) tomando como valor umbral el $95 \%$ del valor modal del histograma de la imagen (Ver Fig. 1(d)). La imagen resultante presenta ruido el cual se elimina aplicando los operadores area opening y area closing (Ver Fig. 1(e)). De esta forma se obtiene una imagen binaria donde 0 representa el fondo de la imagen y 1 representa tejido trabecular.

Etapa 3: En esta etapa se realiza un cambio de espacio color pasando del espacio RGB al HSI para poder realizar el realce. La imagen obtenida en el paso anterior representa el subespacio que caracteriza a las trabéculas. Asignando la tonalidad correspondiente a $120^{\circ}$ a este subespacio en la componente $\mathrm{H}$, se obtiene un realce de los objetos caracterizados por éste, conservando en el resto de la imagen el valor original de la tonalidad (Ver Fig. 1(f)).

El algoritmo fue implementado en MatLab ${ }^{\circledR}$ R2008a, utilizando sus funciones propias y una librería específica de MM denominada SDC Morphology Toolbox [13]. 


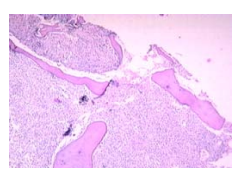

(a)

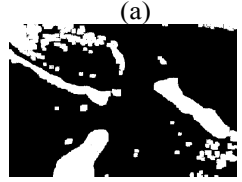

(d)

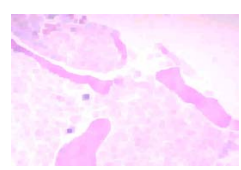

(b)

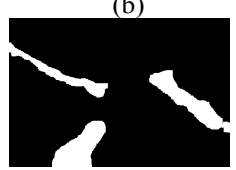

(e)

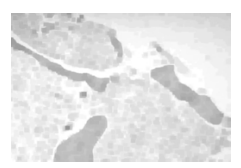

(c)

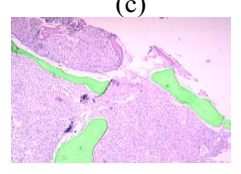

(f)
Figura 1. (a) Imagen original. (b) Imagen resultado del procesamiento color. (c) Componente Verde resultante de la descomposición cromática. (d) Binarización de la componente Verde. (e) Filtrado morfológico Area Opening - Area Closing. (f) Realce en el espacio Color HSI.

\section{Resultados}

A continuación se muestran los resultados que se obtuvieron luego de aplicar el algoritmo presentado.

La Fig. 2 muestra, a modo de ejemplo, los resultados obtenidos en cuatro imágenes de las 30 imágenes procesadas. En la primer columna se muestran las imágenes originales, en la segunda columna se observan las segmentaciones obtenidas a partir del método propuesto $\mathrm{y}$, por último, en la tercer columna se muestra la superposición de la segmentación sobre la imagen original para poder apreciar visualmente los resultados obtenidos.

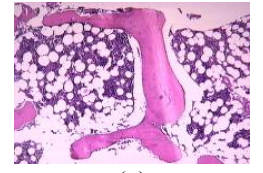

(a)

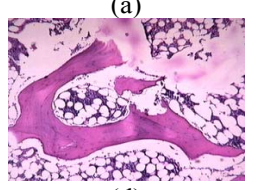

(d)

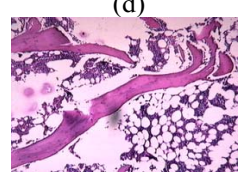

(g)

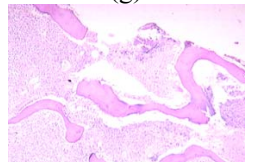

(j)

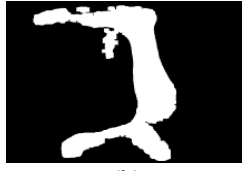

(b)

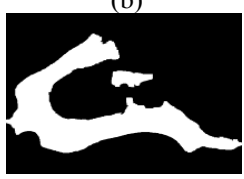

(e)

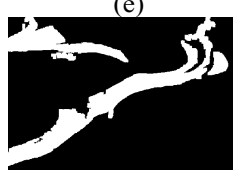

(h)

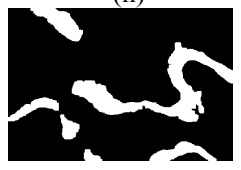

(k)

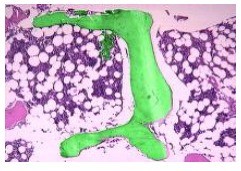

(c)

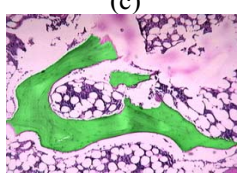

(f)

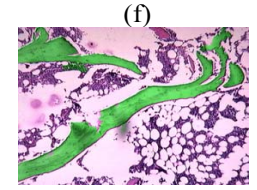

(i)

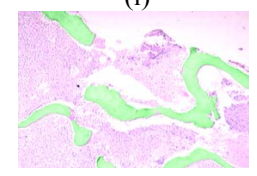

(1)
Figura 1. (a)-(d)-(g)-(j) Imagen original. (b)-(e)-(h)-(k) Segmentación de las trabéculas. (c)-(f)-(i)-(l) Realce en el espacio Color HSI.

\section{V.CONCLUSIONES}

En este trabajo se propuso un método automático para la segmentación de trabéculas en imágenes microscópicas de biopsias de médula ósea, para facilitar su posterior caracterización morfológica. La segmentación de trabéculas en biopsias de médula ósea es un proceso complejo ya que presentan bordes y zonas difusas. Los operadores morfológicos definidos para imágenes color, permitieron lograr una eficiente segmentación facilitando el trabajo del profesional en el diagnóstico médico, disminuyendo la subjetividad del análisis.

En el método propuesto, las imágenes de biopsias de médula ósea fueron modeladas en los espacios color RGB y HSI. En el primer espacio, con el orden lexicográfico, se definieron los operadores básicos de la Morfología Matemática Color, mientras que el espacio HSI se utilizó para realizar el realce de las estructuras trabeculares asignando un falso color en la componente $\mathrm{H}$ al subespacio que representa a las trabéculas.

Luego de realizar las pruebas necesarias y recibir las opiniones de los especialistas, se propone como trabajo futuro implementar una interfaz gráfica de usuario para facilitar la tarea de los expertos.

\section{REFERENCES}

[1] E. Rubin and J. Farber, Patología: Fundamentos, Buenos Aires: Editorial Médica Panamericana, 1992.

[2] B. Frisch, Biopsy Pathology of Bone and Bone Marrow, New York: Raven Press, 1985.

[3] P.G. Bullough, M. Bansal and E.F. DiCarlo, "The tissue diagnosis of metabolic bone disease. Role of histomorphometry", The Orthopedic Clinics of North America, vol. 21, p. 65-79, 1990.

[4] R. Gonzalez and R. Woods, Digital Image Processing, USA: Adison Wesley, 1992.

[5] R. Castleman, Digital Image Processing, Prentice-Hall, Englewood Cliffs, New Jersey, 1979.

[6] D. Ballard and C. Brown, Computer Vision, Prentice Hall, Englewood Cliffs, New York, 1992.

[7] J. Serra, Image analysis and mathematical morphology, Vol. 1, Academic Press, London, 1982.

[8] J. Serra, Image analysis and mathematical morphology, Vol. 2, Academic Press, London, 1988.

[9] J. Facon, Morfología Matemática. Teoría e ejemplos, Editora Universitária Champagnat da Pontífica Universidade Católica do Paraná, Curitiba, Brasil, 1996.

[10] C. Ronse, H. Heijmans, "The algebraic basis of mathematical morphology : II. Openings and closings", Computer Vision, Graphics, and Image Processing: Image Understanding, vol. 54, pp.74-97, 1991.

[11] G. Grätzer, General Lattice Theory, Academic Press, 1978.

[12] A. Hanbury, "Lexicographical Order in the HLS Colour Space", Technical Report W-04/01/MM, Center de Morphologie Mathématique École des Mines de Paris, 2001.

[13] SDC Morphology Toolbox for MATLAB 5. User's Guide. SDC Information Systems, 2001.

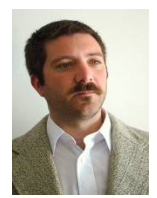

Juan Ignacio Pastore nació en la ciudad de Balcarce, Argentina, en 1977. Obtuvo sus títulos de Profesor en Matemática e Informática Educativa, Profesor en Matemática y Licenciado en Ciencias Matemáticas en la Facultad de Ciencias Exactas y Naturales de la Universidad Nacional de Mar del Plata, en los años 2000, 2001 y 2002, respectivamente. En 2009 obtuvo el título de Doctor en Ingeniería orientación Electrónica. Desde 1998 el Dr. Juan Ignacio Pastore es miembro del Laboratorio de Procesos y Mediciones de Señales, dependiente del Departamento de Electrónica de la Facultad de Ingeniería. Su área de investigación se ha centrado en el Procesamiento de Imágenes aplicando técnicas de Morfología Matemática. 


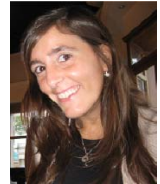

Agustina Bouchet nació en la ciudad de Mar del Plata, Argentina, en 1980. Obtuvo sus títulos de Profesora en Matemática y Licenciada en Ciencias Matemáticas en la Facultad de Ciencias Exactas y Naturales de la Universidad Nacional de Mar del Plata, en los años 2003 y 2005, respectivamente. En 2010 obtuvo el título de Doctora en Ingeniería orientación Electrónica. Desde 2002 trabaja en el Laboratorio de Procesos y Mediciones de Señales, del Departamento de Electrónica de la Facultad de Ingeniería de la Universidad Nacional de Mar del Plata, estudiando el comportamiento de los operadores de la Morfología Matemática Difusa y su aplicación en el Procesamiento de Imágenes Médicas.

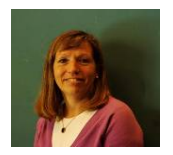

Emilce Moler, nació en la ciudad de La Plata, Argentina, en 1959. Obtuvo su título de Profesora Universitaria en Matemática en la Facultad de Ciencias Exactas de la Universidad Nacional de Mar del Plata en 1983 y su título de Doctora en Ciencias en la Universidad Nacional de Tucumán, en 2003. Desde 1987 es Profesora del Área de Informática Aplicada del Departamento de Matemática de la Facultad de Ingeniería de la Universidad Nacional de Mar del Plata. Desde 1990 es miembro del Laboratorio de Procesos y Mediciones de Señales, del Departamento de Electrónica, donde realiza distintas actividades de investigación, como dirección de proyectos y becarios. Su tema principal de interés es segmentación y representación de Imágenes Biomédicas.

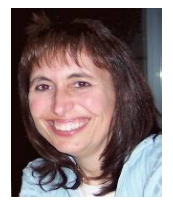

Virginia Laura Ballarin es Profesora Titular Exclusiva e Investigadora categoría I del Departamento de Ingeniería Electrónica de la Universidad Nacional de Mar del Plata. Es actualmente Vice Presidenta de la Sociedad Argentina de Bioingeniería y Directora del Grupo de Procesamiento Digital de Imágenes de la Facultad de Ingeniería de la UNMdP. Recibió su título de Ingeniera Elect. c/o Electrónica y de Magister Scientae de la Universidad Nacional de Mar del Plata y de Doctora en Cs. Biológica orientación Electrónica de la Universidad Nacional de Tucumán. Ha dirigido 11 tesis de posgrado y 18 de Grado y ha publicado más de cincuenta artículos en revistas con referato y más de cien presentaciones en congresos nacionales e internacionales en los tópicos de procesamiento de digital de imágenes especialmente médicas. 